\title{
Contextualiser et augmenter l'attractivité de l'enseignement de base des cir- cuits électriques via l'étude et la réalisation d'un amplificateur audio de classe D
}

\author{
Laurent De Vroey, Stanislas Sobieski,, Francis Labrique, Thierry Daras, Charles Trullemans \\ francis.labrique@uclouvain.be \\ UCL - Département d'Electricité - Place du Levant, 3 - B-1348 Louvain-la-Neuve, Belgique
}

\begin{abstract}
RESUME : En Belgique au niveau de la formation des ingénieurs universitaires, la réforme de Bologne a conduit à remplacer l' approche traditionnelle de deux ans de formation générale suivie de trois ans de spécialisation par une approche de type 3+2 (bachelor degree, master) dans laquelle les étudiants choisissent au cours du premier cycle des modules « techniques », tels que l’électricité, la mécanique, la chimie- physique, ..., les préparant à des masters de spécialisation dans ces domaines. Dans ce contexte où des choix se font très tôt dans le curriculum, offrir via des activités de projet une approche qui permet aux étudiants de découvrir des aspects du métier liés à une discipline peut jouer un rôle significatif au niveau de l'attractivité des formations proposées. C'est dans ce contexte que la présente communication décrit une expérience de projet réalisée cette année et montrant comment l'enseignement des circuits électriques peut permettre aux étudiants de comprendre les principes et réaliser un prototype d'un dispositif en prise directe avec la technologie actuelle, à savoir un ampli audio de classe D.
\end{abstract}

Mots clés : dispositif pédagogique, transfert de savoir-faire, retour d'expérience.

\section{INTRODUCTION}

Les circuits électriques constituent un des éléments de base de la formation des ingénieurs électriciens et électromécaniciens. De leur maîtrise de cet outil dépend en grande partie la facilité avec laquelle les étudiants pourront aborder d'autres matières telles que l'électronique, l'électromécanique ou les télécommunications.

Dans le cadre du passage à la réforme de Bologne et au système 3-5-8 (Bachelor degree, Master degree, Phd, encore appelé LMD en France), l'enseignement des circuits électriques a tendance à se situer de plus en plus tôt dans le curriculum des étudiants. Ainsi à l'Ecole Polytechnique de Louvain la formation aux circuits électriques se situe au deuxième semestre de la deuxième année du $1^{\mathrm{er}}$ cycle et représente une charge de 10 crédits, qui inclut un projet associé à cet enseignement. Cet enseignement s'adresse aux étudiants ayant choisi d'intégrer un module d'électricité dans leur premier cycle. En 2007-2008, ce choix concernait près de 90 étudiants.

Lorsqu'il se situe en début de curriculum l'enseignement des circuits électriques doit se concentrer sur l'acquisition des aptitudes nécessaires à la compréhension physique du fonctionnement des circuits électriques et de leur analyse. Il convient de réserver à des cours situés plus loin dans le curriculum les questions plus avancées de théorie des circuits [1].

Dans l'optique utilitaire qui vient d'être définie, on aboutit au contenu suivant $[2,3]$

- concepts de base (éléments, lois de Kirchhoff, puissances, conservation de la puissance instantanée);

- étude des circuits résistifs (technique de réduction : mise en série, en parallèle, transformation étoile-triangle; équivalents de Norton et de Thévenin);

- étude par les phaseurs de circuits en régime sinusoïdal y compris la conservation de la puissance active et réactive et les circuits triphasés équilibrés;

- réponse en fréquence des circuits en régime sinusoïdal (diagramme de Bode, concept de filtrage);

- $\quad$ étude en régime transitoire de circuits du premier et du deuxième ordre via une approche temporelle;

- $\quad$ analyse des circuits dans le domaine de Laplace;

- $\quad$ étude des quadripôles;

- $\quad$ étude de l'amplificateur opérationnel (y compris certaines de ses imperfections par rapport au modèle idéal) et des montages fondamentaux basés sur ce composant;

- description d'un circuit complexe par une démarche descendante à partir de sa décomposition en blocs fonctionnels.

Une des difficultés de l'enseignement de ces matières se situe au niveau de la perception que les étudiants en ont. Ils trouvent souvent le contenu des cours de circuit trop théorique et peu attractif car ils n'en perçoivent pas l'intérêt. C'est à ce niveau que l'association d'un projet au cours peut intervenir de manière positive en permettant de motiver les étudiants via une contextualisation des matières enseignées. A cet effet, il convient évidemment à veiller à ce que le projet soit attractif et puisse être mené en synergie avec l'enseignement théorique qui le soutient.

Outre la motivation qu'il peut susciter chez les étudiants, associer un projet au cours de circuits permet de répondre à plusieurs objectifs ;

- le premier objectif, déjà mentionné, est de contextualiser des matières telles que la réponse en fréquence, les régimes transitoires des circuits du premier et du deuxième ordre, ou les montages de base des amplificateurs opérationnels;

- $\quad$ un deuxième objectif est d'acquérir une dextérité expérimentale et la maîtrise d'un logiciel 
de simulation de circuits électriques (Spice, par exemple);

- le troisième objectif est de découvrir comment construire sous la forme d'un circuit électrique un dispositif réalisant une fonction donnée et de faire le lien entre un concept et sa matérialisation;

- $\quad$ un quatrième objectif enfin peut être d'identifier le modèle électrique équivalent d'un dispositif électromagnétique ou électromécanique utilisé dans le projet.

Nous avons déjà présenté dans le cadre du Cetsis une première expérience d'association d'un projet au cours de circuits électriques [4]. Dans cette communication, nous allons montrer que prendre comme objet du projet la conception et la réalisation d'un amplificateur audio de classe $\mathrm{D}$ permet de répondre aux objectifs mentionnés ci-dessus, et en particulier à motiver fortement les étudiants (ils pourront brancher leur MP3 sur ce qu'ils auront construit; ils peuvent voir sur le Web les publicités de grands constructeurs pour ce type d'amplificateur) tout en n'exigeant en matière de coût de composants qu'un investissement raisonnable si on tient compte qu'on peut faire travailler les étudiants par groupe de 6 en leur demandant de comparer deux solutions, chacune traitée par un groupe de 3 .

\section{PRESENTATION DU PROJET. DESCRIP- TION CONCEPTUELLE DU DISPOSITIF A REALISER}

La présentation d'un projet constitue une étape essentielle car de son contenu dépendra la manière dont les étudiants entreront dans le projet. Il est primordial de susciter d'entrée de jeu la motivation qui est la condition nécessaire à l'apprentissage. Dans le cadre de la réalisation d'un amplificateur audio de classe $\mathrm{D}$, la présentation a commencé en faisant référence à des sites internet publicitaires présentant des amplificateurs pour audiophiles. Ensuite, elle est entrée dans les aspects techniques en parcourant les trois étapes décrites cidessous.

\subsection{Principe de la modulation de largeur d'impul- sion}

Dans le cadre de la réalisation d'un amplificateur audio de classe $\mathrm{D}$, la première étape de la description conceptuelle du dispositif à réaliser revient à introduire et faire comprendre le concept de la modulation de largeur d'impulsion ou MLI.

La figure 1 synthétise le principe de la modulation de largeur d'impulsion : transformer un signal $v_{x}$ pouvant varier d'une valeur $V_{\min }$ à une valeur $V_{\max }$ (par exemple de $-15 \mathrm{~V}$ à $+15 \mathrm{~V}$ ) en un signal périodique en créneaux prenant alternativement les valeurs $V_{\min }$ et $V_{\max }$ avec sur chaque période un temps de présence à $V_{\max }$ et un temps de présence à $V_{\min }$ tels que la valeur moyenne soit égale à $v_{x}$.

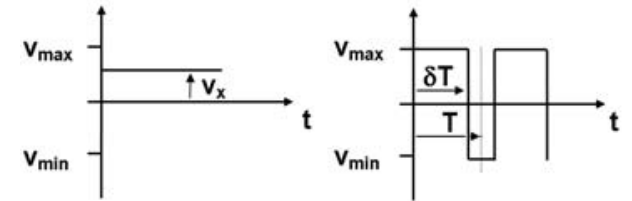

fig 1 : Principe de la modulation de largeur d'impulsion (MLI)

On veut avoir :

$<v_{M L I}>=\frac{1}{T_{M L I}}\left[\int_{0}^{\delta T_{M L I}} V_{\max } d t+\int_{\delta T_{M L I}}^{T_{M L I}} V_{\min } d t\right]=v_{x}$

soit :

$<v_{M L I}>=\delta V_{\max }+(1-\delta) V_{\min }=v_{x}$

Si $V_{\max }=-V_{\min }=\mathrm{V}$, la relation précédente devient :

$(2 \delta-1) V=v_{x} \quad$ soit $\quad \delta=\frac{1}{2}+\frac{1}{2} \frac{v_{x}}{V}$

A ce stade, on a demandé aux étudiants d'analyser le signal MLI par une décomposition en série de Fourier (lien avec les cours de mathématique). Ils ont ainsi perçu que le signal MLI contient

- une composante utile : sa valeur moyenne égale à $v_{x}$;

- des composante parasites : ses termes sinusoïdaux à la fréquence MLI et ses multiples.

\subsection{Comment produire le signal MLI}

La deuxième étape de la présentation conceptuelle du système à réaliser a consisté à analyser deux manières dont on peut produire un signal MLI de valeur moyenne $v_{x}$

- la première méthode appelée la modulation intersective (conceptuellement la plus simple) revient à comparer la valeur du signal $v_{x}$ à un signal auxiliaire (appelé la porteuse) en triangle ou en dent de scie oscillant à une fréquence fixe entre deux niveaux égaux à $V_{\min }$ et $V_{\max }$ et à imposer au signal MLI de valoir $V_{\max }$ lorsque le signal $v_{x}$ a une valeur supérieure à la porteuse et $V_{\min }$ dans le cas contraire (figure 2);

- la deuxième méthode est la modulation sigma delta $(\Sigma-\Delta)$ : elle consiste à intégrer l'écart existant entre le signal MLI $v_{M L I}$ et le signal $v_{x}$ et à maintenir ce signal entre deux butées $v_{+}$et $v_{\text {. en forçant }} V_{M L I}$ à passer de $V_{\max }$ à $V_{\min }$ lorsque le signal intégral atteint $v_{+}$en croissant et de $V_{\min }$ à $V_{\max }$ lorsque le signal intégral atteint $v$. en décroissant (figure 3 ).

A ce stade les étudiants ont pu vérifier que les deux méthodes permettent d'obtenir un signal MLI dont la valeur moyenne correspond à $v_{x}$. Ils ont pu également constater que la modulation $\Sigma-\Delta$ conduit à une modulation dont la fréquence varie en fonction de la valeur $v_{x}$ et que si on veut obtenir un signal MLI dont la fréquence ne descend pas en-dessous d'une valeur 


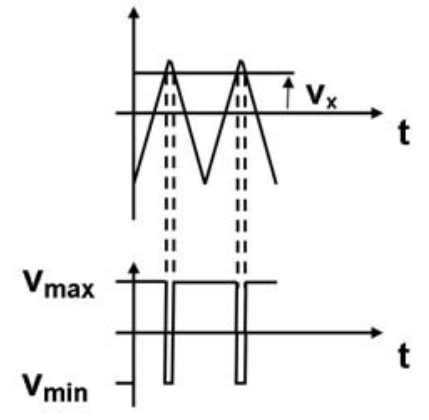

fig 2 : Principe de la modulation intersective

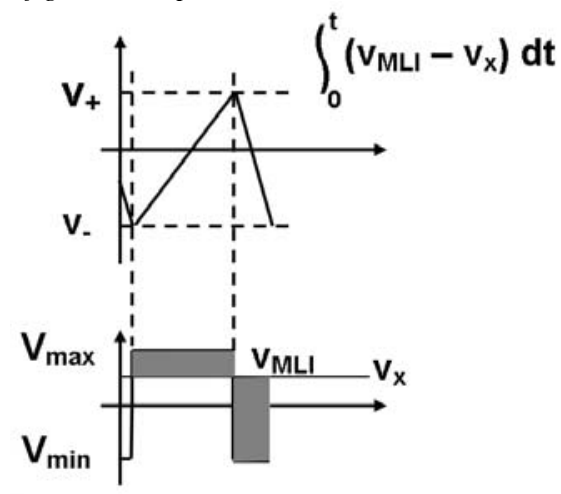

fig 3 : Principe de la modulation sigma-delta

minimum imposée, il faut limiter la plage de valeur admissible pour $v_{x}$. Dans les cas où $V_{\max }=-V_{\min }=V$, la figure 4 montre pour la modulation $\Sigma$ - $\Delta$ l'évolution en fonction de $v_{x}$ :

- du temps $T_{+}$durant lequel le signal MLI est à $+V$ :

$$
T_{+}=\frac{\left(v_{+}-v_{-}\right) / V}{1-v_{x} / V}
$$

- du temps $T$. durant lequel le signal MLI est à $-V:$

$$
T_{-}=\frac{\left(v_{+}-v_{-}\right) / V}{1+v_{x} / V}
$$

- $\quad$ de la fréquence MLI :

$$
f=\frac{1}{T_{+}+T_{-}}=\frac{1-\left(v_{x} / v\right)^{2}}{\left(v_{+}-v_{-}\right) / V}
$$

\subsection{Architecture de l'amplificateur}

La figure 5 donne le schéma de principe de l'amplificateur MLI dans le cas où $V_{\max }=-V_{\min }=\mathrm{V}$.

- le bloc prétraitement du signal assure une pré-amplification et un filtrage de type passe bas du signal à amplifier( nous avons choisi d'imposer à ce bloc de fournir en sortie un si gnal d'amplitude de 5 volt crête au maximum pour une entrée de $300 \mathrm{mV}$ et d'avoir une fréquence de coupure de $3.3 \mathrm{KHz}$ conforme aux standards de la téléphonie);

- $\quad$ Le bloc modulateur MLI assure la transformation du signal $v_{x}$ en signal MLI.
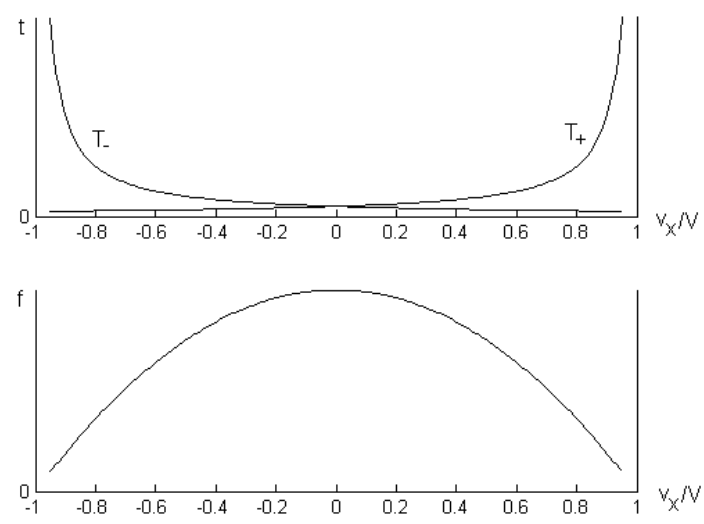

fig 4 : Evolution des temps $T_{+}$et $T_{-}$de signal $+V$ et $-V$ (haut) et de la fréquence MLI (bas)

- l'étage de puissance comporte deux interrupteurs (formés à l'aide de semiconducteurs de puissance) permettant de relier la charge soit à une source de valeur $+\mathrm{V}$ ou à une source de valeur $-\mathrm{V}$ en fonction de l'interrupteur qui est fermé $\left(\mathrm{S}_{+}\right.$ou $\left.\mathrm{S}_{-}\right)$. Il reproduit le signal MLI car l'état des interrupteurs est fixé par ce signal :

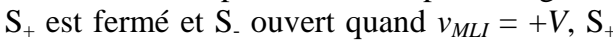
est ouvert et $\mathrm{S}$. fermé quand $v_{M L I}=-V$. Ce bloc peut délivrer à la charge un courant dont la valeur maximum est fixée par le courant que les interrupteurs peuvent supporter. Le rendement de cet étage est proche de $100 \%$ dans la mesure où la chute de tension aux bornes d'un interrupteur fermé est virtuellement nulle de même que l'est le courant qui traverse un interrupteur ouvert;

- le bloc filtre de reconstruction + charge contient un filtre dit de reconstruction qui atténue fortement les composantes parasites engendrées par la MLI de sorte qu'aux bornes de la charge proprement dite (le haut-parleur que l'ampli alimente) on retrouve essentiellement la composante utile du signal MLI (celle qui est l'image de $v_{x}$ ).

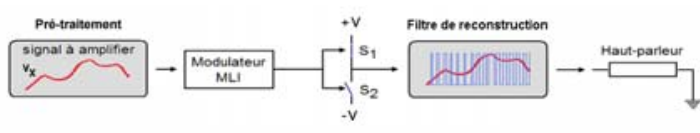

fig 5 : Architecture de l'amplificateur

A ce stade, en supposant que la charge est purement résistive et que le filtre de reconstruction se limite à une simple inductance $L$, les étudiants peuvent si on leur donne la valeur de $R_{c h}$, et une contrainte à respecter sur la fréquence MLI :

- calculer la valeur de l'inductance $L$ pour que la fréquence de coupure du circuit formé par $L$ et $R_{c h}$ soit de $3.3 \mathrm{kHz}$; 
- calculer pour une MLI intersective le courant absorbé par la charge en fonction de la valeur de $v_{x}$ (supposée constante) en utilisant soit une analyse fréquentielle, soit un calcul basé sur la réponse transitoire de la charge à un créneau et vérifier que la tension aux bornes de $R_{c h}$ est essentiellement fixée par $v_{x}$;

- calculer dans le cas de la modulation $\Sigma-\Delta$ l'hystérèse $\left(v_{+}-v_{-}\right) / V$ pour que la fréquence MLI ne dépasse pas une valeur maximum donnée et calculer la dynamique d'amplitude admissible pour $v_{x}$ pour que la fréquence MLI ne descende pas en-dessous d'une valeur minimum donnée ;

- $\quad$ comparer en fonction de la valeur de $v_{x}$ la tension obtenue au niveau de $R_{c h}$ avec la modulation $\Sigma$ - $\Delta$ par rapport à la modulation intersective.

\section{REALISATION DE LA CELLULE DE BASE DU MODULATEUR}

\subsection{Analyse, simulation en Spice et réalisation au laboratoire de montages élémentaires à amplifica- teurs opérationnels}

La prise de contact avec le laboratoire s'est faite par l'étude de montages simples nécessitant la prise en main des principaux appareils. L'étude d'un filtre passe-bas de premier ordre, au moyen d'une cellule RC puis d'un montage à amplificateur opérationnel, a permis aux étudiants de manipuler le matériel expérimental: plaquettes de tests (breadboards), amplificateurs opérationnels composants passifs, alimentation DC, générateur de signaux. Ils se sont familiarisés également aux appareils de mesure : multimètre, oscilloscope, ainsi qu'aux techniques qui y sont liées : notion de gain d'une sonde d'oscilloscope, affichage AC/DC, mesure de déphasage, raccord des masses du circuit et des appareils,...

Cette étape de prise en main a été fortement dirigée, un maximum d'informations et conseils utiles étant donnés d'emblée aux étudiants par les enseignants. Ceci a permis de gagner un temps précieux par la suite, l'attention étant attirée dès le départ sur certaines erreurs grossières à éviter; une utilisation imprudente des sondes d'oscilloscopes, par exemple, enfoncer profondément leur pointe dans les plaquettes de test, peut conduire à un écartement permanent des contacts internes, résultant en la présence de capacités parasites perturbant sensiblement les mesures.

L'approche de montages simples a permis également une prise en main du logiciel de simulation PSpice; les étudiants se sont familiarisés ainsi à la comparaison critique des résultats théoriques et expérimentaux, ainsi qu'au dimensionnement de circuits.

\subsection{Structure et dimensionnement d'une cellule de base du modulateur}

Les principaux outils étant connus, les étudiants ont pu se concentrer sur le coeur du projet, que constitue la cellule de base du modulateur. Un schéma PSpice leur a été fourni (figure 6), dont les principes de fonctionnement ont été évoqués au cours théorique ainsi que dans le cadre de séances de travaux dirigés.

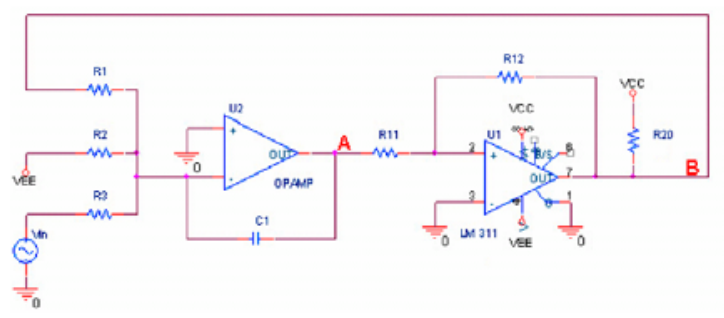

fig 6 : Cellule de base du modulateur MLI

Ce schéma, selon que la source $V_{\text {in }} \mathrm{y}$ soit ou non raccordée, peut respectivement représenter un modulateur $\Sigma$ - $\Delta$ (avec sortie en $\mathrm{B}$ ) ou un générateur de tension triangulaire pouvant servir à créer une porteuse (à partir de la sortie en A). Les valeurs des composants ne sont pas fournies, mais des contraintes sont imposées aux étudiants quant à la fréquence MLI maximale souhaitée $(50 \mathrm{KHz})$, l'amplitude du signal souhaité au point $\mathrm{A}$ et le courant maximum que peut fournir l'amplificateur opérationnel. A ce stade, et c'est volontaire, les étudiants sont amenés à découvrir que le comparateur utilisé est du type "open collector" et qu'après placement d’une résistance de "pull up" ce circuit fournit une tension de sortie égale à zéro ou $+\mathrm{V}$ et non égale à $-\mathrm{V}$ ou $+\mathrm{V}$ comme c'était le cas dans la présentation faite à la section 2 . Il faut alors leur faire comprendre que cela ne pose pas un réel problème car

- $\quad$ un signal oscillant entre 0 et $+\mathrm{V}$ peut être vu comme un signal oscillant entre $-\mathrm{V} / 2$ et $+\mathrm{V} / 2$ à une composante continue $\mathrm{V} / 2$ près et que cette composante peut être compensée vis-àvis de l'intégrateur par une entrée supplémentaire (fournie par la borne négative de la source d'alimentation $\left(\mathrm{V}_{\mathrm{EE}}=-\mathrm{V}\right)$ à travers une résistance $R_{2}$ de valeur bien choisie);

- rien n’oblige les valeurs $v_{+}$et v. d'être symétriques par rapport à zéro : avec le comparateur à hystérésis choisi, elles valent 0 et $\left(\mathrm{R}_{11} / \mathrm{R}_{12}\right) \mathrm{V}_{\mathrm{B}}$.

Il faut également leur faire comprendre que la fréquence MLI ne dépend pas seulement des seuils de basculement fixés par le comparateur à hystérésis mais qu'on peut aussi agir sur sa valeur via les valeurs de $\mathrm{R}_{1}$ et $\mathrm{C}_{1}$. Ces points sont importants car ils montrent comment la réalisation peut imposer des contraintes ou offrir des degrés de liberté par rapport au concept de départ sans altérer la fonctionnalité du produit réalisé. 
Le travail demandé aux étudiants a été de dimensionner et d'étudier ce montage par simulation, calculs et mesures, pour les deux cas de figure évoqués :

- $\quad$ utilisation en modulateur sigma-delta;

- génération d'une porteuse triangulaire ( il faut alors adapter l'amplitude de $\mathrm{V}_{\mathrm{A}}$ et éliminer la composante continue qui y est présente par exemple via un filtre passe haut.

Ceci a donné lieu à un premier rapport, dont l’objectif est principalement de pousser les étudiants à clarifier leurs idées et à pouvoir les présenter de manière critique et rationnelle. Ce rapport a donné lieu à un "débriefing" en deux étapes :

- en premier lieu un "débriefing" global où les problèmes les plus fréquemment rencontrés ont été analysés (manque de logique dans le plan, incapacité d'exprimer de manière simple (par décomposition du problème), les relations fondamentales pour le dimensionnement,...);

- en second lieu un entretien individuel avec chaque groupe pour traiter les problèmes spécifiques au groupe et orienter les étudiants pour la suite du travail.

Les étudiants avaient dimensionné les éléments en écrivant suffisamment d'équations pour pouvoir déterminer les valeurs inconnues, mais sans prendre la peine de structurer le système en blocs et de nommer les signaux d'interface entre les blocs. Par exemple, si à la figure 6 on considère l'ensemble R1, R2, R3 comme un sommateur de courant, et son courant de sortie comme le signal d'entrée d'un intégrateur, on peut écrire :

$$
V_{A}=\frac{1}{C} \int\left(\frac{1}{R_{1}} V_{B}+\frac{1}{R_{2}} V_{E E}+\frac{1}{R_{3}} V_{\text {in }}\right) d t
$$

Cette expression présente clairement beaucoup d'information à propos du fonctionnement du montage.

\section{MONTAGE FINAL ET TESTS}

Le montage final s'articule autour du bloc modulateur étudié au point précédent suivant le schéma de la figure 5. Les autres éléments nécessaire (étage de conditionnement à l'entrée, étage de puissance , filtre de reconstruction) sont fournis aux étudiants. Il leur est demandé d'en comprendre le rôle et d'être capables de les dimensionner, mais pas de les réaliser.

L’étage de puissance peut être réalisé de manière simple à l'aide d'un circuit intégré spécialisé par exemple le LMD18200 de National qui comporte un pont en $\mathrm{H}$ dont l'état des interrupteurs peut directement être commandé par un signal logique obtenu en écrêtant la sortie $\mathrm{V}_{\mathrm{B}}$ du comparateur pour la ramener entre zéro et $5 \mathrm{~V}$ à l'aide par exemple du montage de la figure 7.

Deux utilisations de ce circuit sont possibles. La première (figure 8a) consiste à placer le haut parleur et le filtre de reconstruction (formé d'une simple inductance en série avec lui) dans la branche horizontale du pont de manière à appliquer à cet ensemble $+\mathrm{V}$ quand la sortie du modulateur est au niveau haut et $-\mathrm{V}$ lorsque

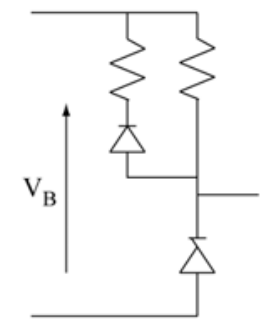

fig 7 : circuit de passage à un niveau TTL

cette sortie est au niveau bas. La deuxième (figure 8b) consiste à placer le haut parleur et le filtre de reconstruction entre la sortie d'un bras et la masse. Dans ce cas le signal appliqué au haut parleur et au filtre vaut $+\mathrm{V}$ quand la sortie du modulateur est au niveau haut et 0 quand cette sortie est au niveau bas. Le signal de puissance comporte alors une composante continue égale à V/2 qu'on élimine en incluant dans le filtre une capacité en série avec l'inductance, la valeur de cette capacité étant choisie de manière à avoir vers le bas une fréquence de coupure à $300 \mathrm{~Hz}$ (à nouveau en accord avec les standards de la téléphonie). Lorsqu'on adopte cette solution il est intéressant de faire remarquer aux étudiants la différence de démarche adoptée pour supprimer la composante continue par rapport à ce qui a été fait au niveau du modulateur.
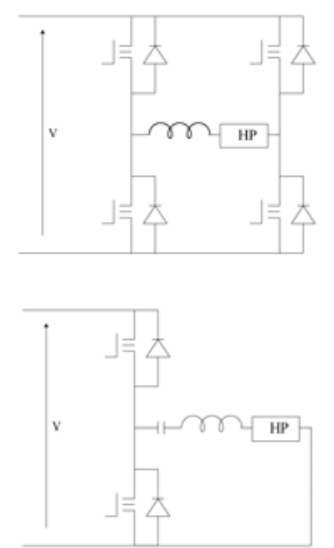

fig 8: Configurations possibles de l'étage de puissance

Chaque groupe a travaillé sur les deux montages MLI intersective et $\Sigma-\Delta$, ce qui leur a permis d'en comparer les principes et résultats, notamment en termes de variation temporelle des signaux MLI et en termes d'oscillation des signaux résultants. On leur a demandé d'effectuer des mesures en différents points en expliquant pourquoi les signaux obtenus ont telle ou telle forme et pourquoi éventuellement ils s’écartent de ceux fournis par la simulation. On a demandé notamment de visualiser la différence entre entrée et sortie générales du montage. 
Cerise sur le gâteau, une évaluation qualitative a été proposée grâce au branchement d'un lecteur MP3 en entrée, qui permet aux étudiants d'apprécier le rendu de leurs montages au haut-parleur.

Le travail s'est achevé par la rédaction d'un rapport synthétique, partant d'une version révisée du rapport intermédiaire présenté précédemment et complété par les résultats ultérieurs, ainsi que par la présentation en laboratoire des résultats expérimentaux et de simulation. La lecture de ces rapports a permis de vérifier qu'en moyenne le projet avait répondu aux attentes, les notes de groupes se situant entre 11 et 16 avec une moyenne autour de 14.

\section{DEROULEMENT DETAILLE DU PROJET}

Ce projet se déroule sur 14 semaines. L’équipe d'encadrement se réunit chaque semaine pour piloter le déroulement du projet et déterminer le support qui sera apporté aux étudiants en fonction de l'état d'avancement observé (documents, consultance, sousensembles pré câblés...). Les 90 étudiants sont répartis par groupe de 6 formés de manière arbitraire. Chaque groupe a 2 heures par semaine en laboratoire encadrées par 2 ATER et 2 étudiants moniteurs. Cet encadrement concerne 40 à 50 étudiants par séance de 2 heures. Un travail non encadré, individuel ou en groupe, de 2 à 4 heures par semaine est attendu de la part des étudiants. Sur toute la durée du projet, les étudiants sont dans un premier temps guidés avec des objectifs simple, en ayant toujours à l'esprit l'objectif final, pour arriver en fin de projet à une autonomie accompagnée. Dans le même sens, les informations qui leur sont fournies en début de projet sont multiples.

En outre, en parallèle, durant les séances de TP du cours de circuits (une séance de $2 \mathrm{~h}$ par semaine), une partie des exercices proposés visent à donner des pistes pour résoudre les problèmes posés par le projet.

Ce projet se situant en début de curriculum en génie électrique, les étudiants concernés sont encore peu ou pas formés à la manière de gérer efficacement une activité de laboratoire (TP) qui ne consiste pas à effectuer une manipulation déterminée à l'avance.. A raison de $14 \times 2 h$ en laboratoire, il est donc nécessaire d'être directif au niveau des consignes initiales de mise en place du projet.

De manière détaillée, le projet s’est déroulé comme suit:

- S1 : présentation du projet et formation des groupes;

- S2-S5 : nous avons organisés des séances de prise en main des outils de mesure et de simulation de circuits simples, tout en favorisant l'auto-apprentissage. Ces circuits, tel que l'intégrateur et la bascule à hystérèse, correspondent à des modules qui seront utilisés dans le circuit complet;

- S6 : dernière séance de prise en main sur un circuit correspondant à la cellule de base du modulateur MLI avec un dimensionnement imposé. Les étudiants doivent réaliser un dimensionnement de cette cellule en accord avec les spécifications du projet données en $\mathrm{S} 1$ et en effectuer la simulation;

- S7-S10 : montage et mesure de la cellule de base du modulateur MLI. On leur demande en outre de concevoir, sur base des blocs fonctionnels du modulateur $\Sigma$ - $\Delta$, un modulateur basé sur la méthode intersective;

- $\quad$ S11 : intégration de la cellule MLI aux autres éléments de l'amplificateur qui sont fournis mais dont on demande aux étudiants d'être capables d'analyser le fonctionnement et test du système complet (amplification d'un signal provenant d'un lecteur mp3);

- S12 : évaluation des performances des montages finaux;

- S13 : dernières mesures et rédaction du rapport final;

- $\quad$ S14 : présentation et démonstration de leur réalisation par chaque groupe.

\section{CONCLUSION}

Cette communication montre comment on peut contextualiser l'enseignement de base des circuits électriques via un projet aussi ambitieux que la réalisation d'un amplificateur audio de classe D. Nous avons indiqué comment, outre son caractère motivant, ce projet permettait de répondre aux objectifs mentionnés dans l'introduction. Nous espérons enfin que l'expérience rapportée pourra être utile à d'autres équipes d'enseignants.

\section{REMERCIEMENTS}

Les auteurs expriment leur gratitude aux membres de l'équipe d'encadrement, François Baudart, Olivier Bulteel, Thierry Daras, Jonathan Denies, Julien Devos, Thibaut Labbé, Christophe Mouvet, Paul Sente et Christophe Vloebergh et aux étudiants qui, par leur participation active, ont contribué à façonner ce projet.

\section{Bibliographie}

[1] Marc Fosséprez, "Topologie et comportement des circuits non linéaires non réciproques", Presses Polytechniques Romandes, 1989, ISBN: 2-88074165-3.

[2] James W. Nilson, "Electric circuits", AddisonWesley, 1993, ISBN : 0-201-54987-5.

[3] David E. Johnson, Johnny R. Johnson, John L. Hilburn, Peter D. Scott, "Electric Circuits Analys", Prentice-Hall, 1997, ISBN : 013398074-X.

[4] F. Vrins, L. De Vroey, F. Labrique, C. Trullemans, "Apprentissage par projet en électricité (partie 1) : Conception et réalisation d'un système de filogui- 
dage électromagnétique", Proceedings du Congrès CETSIS-EEA 2003, pp. 431-434. 